\title{
Simulation of HyMETS Flowfield around Baby-SPRITE Entry Probe
}

\author{
Patricia Ventura Diaz, ${ }^{*}$ Seokkwan Yoon, ${ }^{\dagger}$ Francesco Panerai ${ }^{\ddagger}$ and Nagi N. Mansour ${ }^{\dagger}$ \\ NASA Ames Research Center, Moffett Field, California
}

\begin{abstract}
Arc-jets are unique facilities used in research, development, and evaluation of hightemperature thermal protection systems for hypersonic vehicles and planetary entry systems. Thermochemical non-equilibrium computational fluid dynamics simulations have been carried out for the Hypersonic Materials Environmental Test System arc-jet facility to determine the size of a capsule model before arc-jet testing by better understanding of the physical phenomena. The results show the effect of the test article geometry and the importance of high-quality grids for accurate solutions. Accurate computational modeling of hypersonic flow fields inside arc-jets under simulated planetary entry conditions would help improve the design of thermal protection systems that may enable human exploration of the Moon, Mars, and beyond.
\end{abstract}

\section{Introduction}

The Hypersonic Materials Environmental Test System, HyMETS, is a $400 \mathrm{~kW}$ arc-jet wind tunnel housed at NASA Langley Research Center in Hampton, Virginia. The facility provides a ground test environment that simulates aerothermal loads and pressures typical of high-speed entry into planetary atmospheres. HyMETS is primarily used for screening Thermal Protection System (TPS) materials at a range of conditions relevant to hypersonic planetary entry. Compared to large scale arc-jets, this facility provides the ability to test a large number of samples with a very short turnaround time and inexpensive operation by a single technician.

In this work, the HyMETS environment is simulated using aerothermal Computational Fluid Dynamics (CFD). The effort was carried out in support of a forthcoming test campaign on the Phenolic Impregnated Carbon Ablator, PICA, the lightweight TPS material used for the Mars Science Laboratory (MSL) heatshield. The PICA fore-surface of the MSL TPS assembly was covered with a thin overcoat of silicone material, designated NuSil CV-1144-0 (NuSil Technology LLC, Carpinteria, CA, USA). NuSil was applied to hinder particulate shedding from PICA's phenolic, as a contamination control measure during clean-room Assembly, Test, and Launch Operations (ATLO).

Current knowledge on how NuSil affects the response of PICA under Mars entry conditions is very limited. Past analyses of flight data collected by the MSL Entry Descent and Landing Instrumentation (MEDLI) suite did not account for NuSil-related effects. As MEDLI data have been extensively used to assess the performance of state-of-the-art hypersonic CFD tools and ablator response models for PICA, it is critical to study the behavior of NuSil under entry conditions and its effects, if any, on the high-temperature response of PICA. A dedicated research effort is currently underway at NASA to understand the response of the PICA-NuSil (PICA-N) system, in support of Mars 2020 mission.

A test campaign has been planned at the HyMETS facility to screen the response of NuSil-coated PICA and gather detailed data on the behavior of PICA-N under simulated Mars entry conditions. For the campaign a test model has been designed, inspired by the Small Probe Re-entry Investigation of TPS Engineering (SPRITE) design, developed by Prabhu at NASA Ames Research Center. ${ }^{1}$ Prabhu's SPRITE design is a full-scale $355 \mathrm{~mm}$ (14 in) diameter entry probe. It was tested in the 18-inch nozzle of the

*Science and Technology Corporation.

${ }^{\dagger}$ NASA Advanced Supercomputing Division.

${ }^{\ddagger}$ University of Illinois at Urbana-Champaign. 
Aerodynamic Heating Facility (AHF), one of NASA Ames Research Center's arc-jets. In this work we study a smaller SPRITE model, that we refer to as baby-SPRITE.

High-fidelity CFD can predict the flowfield and help understand the physics of atmospheric entry. Variables such as heat transfer coefficient, boundary layer thickness, boundary layer edge enthalpy, and edge Mach number, which are essential to characterize TPS and cannot be captured with ground tests, can be predicted with CFD. However, there are many computational challenges in hypersonic flow simulations, and they have to be addressed carefully in order to accurately predict the flow. ${ }^{2}$ The complex physics and the wide range of spatial and time scales present in hypersonic flows, which contain gas-surface interactions, entropy and shear layers, shock waves, and real gas effects, make the development of efficient and accurate numerical simulation methods extremely challenging. Some of the challenges include the accurate simulation of turbulence due to roughness-induced transition, reliable hybrid RANS-LES methods, grid adaptation to capture essential flow features, and multi-physics coupling.

Previous computational work on the HyMETS arc-jet facility includes the studies by Brune et al. ${ }^{3}$ and Duzel et al. ${ }^{4}$ The work done by Brune et $a l^{3}{ }^{3}$ proposed an alternative method to estimate the flightrelevant heat flux and heat load, and predict the relationship between the flight and arc-jet surface heat flux by direct simulation of the arc-jet facility using NASA's CFD code LAURA-5. Duzel et al. ${ }^{4}$ showed the computational results of a single test case that consisted of $6.5 \mathrm{MJ} / \mathrm{kg}$ enthalpy flow that discharged into the vacuum chamber at $0.228 \mathrm{kPa}$, and compared these results to experiments.

The purpose of this work is to simulate the HyMETS flowfield around different geometries in order to 1) refine and verify the design of the test geometry for the HyMETS facility, and 2) compute aerothermal environment inputs for material response simulations of PICA-NuSil. Initially, the $33 \mathrm{~mm}$ (1.3 in) diameter cold-wall calorimetric probe used at HyMETS to calibrate test conditions is simulated. A first baby-SPRITE design is then simulated. From the converged CFD solution, the design of the initial baby-SPRITE is changed to a smaller base diameter in order to avoid flow blockage. The importance of having a high-quality grid to obtain accurate flowfield solutions is highlighted. In addition, the computational solution has been used to assist in the preparation of new test geometries.

\section{HyMETS Facility and Baby-SPRITE Test Model}

The HyMETS facility is a segmented direct current electric arc-heater. The plasma generator consists of a series of water-cooled elements, including a copper cathode with a tungsten button emitter, a $12.7 \mathrm{~mm}$ (0.5 in) bore diameter column of 32 copper segment constrictors, and a copper divergent-ring anode. The flow is accelerated to Mach 5 through a copper convergent-divergent 8 degree half-angle nozzle, with a 12.7 $\mathrm{mm}(0.5 \mathrm{in})$ throat diameter and a $63.5 \mathrm{~mm}(2.5 \mathrm{in})$ exit plane diameter.

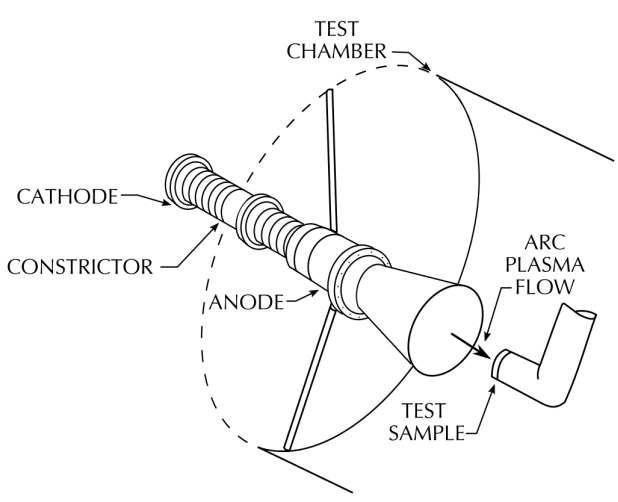

(a) HyMETS facility test setup schematic.

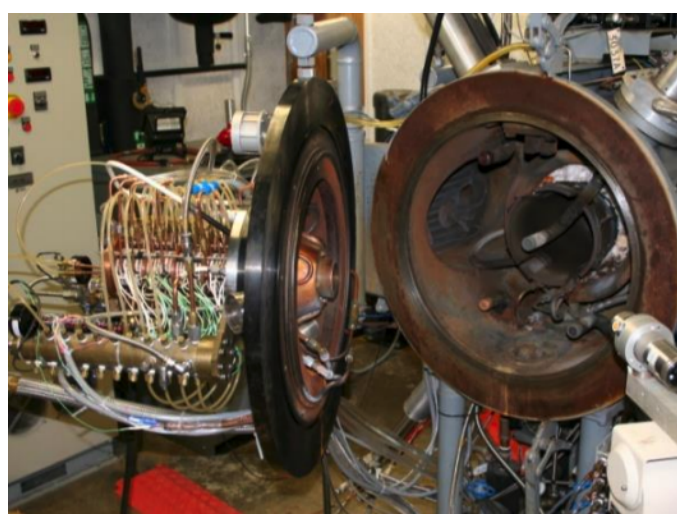

(b) HyMETS facility setup.

Figure 1: The HyMETS facility.

The $60 \mathrm{~cm}$ diameter $(2 \mathrm{ft}), 90 \mathrm{~cm}(3 \mathrm{ft})$ long test chamber houses four piston-controlled water-cooled stings that can be inserted into the plasma jet on demand. Three stings mount flow calibration probes: a copper slug and a Gardon gauge calorimeter for cold-wall fully-catalytic heat flux measurements and a Pitot probe for stagnation pressure. The fourth probe mounts the material sample test model. Calibration probes 
sit at a standard location, $50.8 \mathrm{~mm}$ downstream of the nozzle exit, while the sample probe location depends on the geometry of the test model.

The flow is exhausted through a $20.32 \mathrm{~cm}$ (8 in) inlet diameter collector cone, followed by a $15.24 \mathrm{~cm}(6$ in) diameter constant cross-section diffuser. Downstream of the diffuser the flow is cooled through a coiled copper tubing heat exchanger prior to discharging into the mechanical pumping system.

The facility operates using a range of test gases including argon, oxygen, nitrogen, and carbon dioxide, injected tangentially at discrete locations of the arc column. Gas mixtures are regulated by mass flow controllers, enabling simulations of Earth and Mars entry plasmas at heat fluxes up to $\approx 500 \mathrm{~W} / \mathrm{cm}^{2}$ and stagnation pressures of $\approx 9 \mathrm{kPa}$.

Further details on the facility and its operating envelope are documented in the work by Splinter et al. ${ }^{5}$

The baby-SPRITE test model designed for the PICA-N test campaign is shown in Figure 2. It features a SPRITE-shaped $55^{\circ}$ sphere-cone PICA shell that houses a thermocouple plug for real-time material temperature measurements during arc-jet exposure.

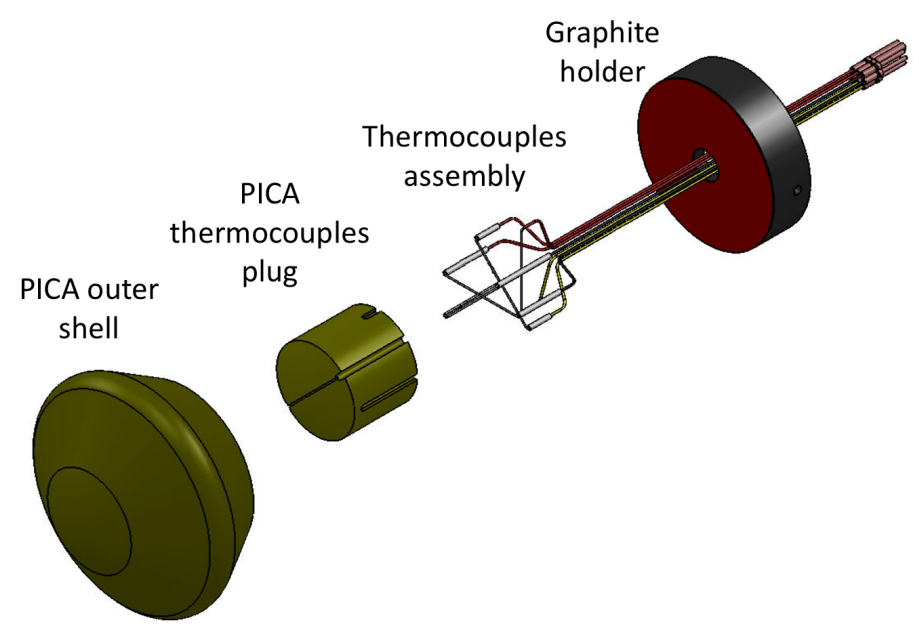

Figure 2: Schematic of the baby-SPRITE model assembly.

Arc-jet facility dimensions, in particular the nozzle and diffuser diameters, place an upper bound on the size of the test specimen because of flow blockage. The primary consideration in flow blockage is the inability of the diffuser to capture the supersonic mass flow.

The HyMETS facility has a relatively small nozzle and diffuser diameter. The new design of the babySPRITE has never been tested in HyMETS. A simpler approach than build-and-test, is to run CFD simulations to determine if the baby-SPRITE design will allow the mass flow to be captured by the diffuser.

\section{Geometry Modeling and Grid Generation}

A sketch of the HyMETS facility described in the previous section is shown in Figure 3. The plasma flow from the arc-jet is accelerated in the nozzle and exhausted into the test chamber. In this study, two specimens are simulated inside the test section: the cold-wall calorimetric probe, and the PICA-NuSil baby-SPRITE.

The geometry of the calorimetric probe is illustrated in Figure 4a. The diameter is $33 \mathrm{~mm}$ (1.3 in), and the radius at the edge is $3 \mathrm{~mm}(0.15 \mathrm{in})$. The initial design of the baby-SPRITE can be seen in Figure $4 \mathrm{~b}$. It is a $55^{\circ}$ sphere-cone with a $60 \mathrm{~mm}(2.371 \mathrm{in})$ base diameter. The nose radius is $17 \mathrm{~mm}(0.705 \mathrm{in})$. The radius of the shoulder is $3.8 \mathrm{~mm}(0.150 \mathrm{in})$. The conical frustum of the aft shell has a $30^{\circ}$ inclination to the horizontal. The initial geometry of the baby-SPRITE appeared to be too big and too close to the nozzle exit plane (NEP), possibly leading to flow blockage in the facility, and so CFD simulations were required before the test campaign. Indeed, the first proposed geometry of baby-SPRITE was too big, and CFD simulations revealed unsteady shock-plume interaction in addition to supersonic mass flow overflowing the diffuser, which can damage the arc-jet facility. The final baby-SPRITE geometry is shown in Figure 4c. The base diameter has been reduced to $46 \mathrm{~mm}$ (1.828 in).

For CFD analysis, i.e., the prediction of surface aerothermal environments (pressure, heat flux, and 


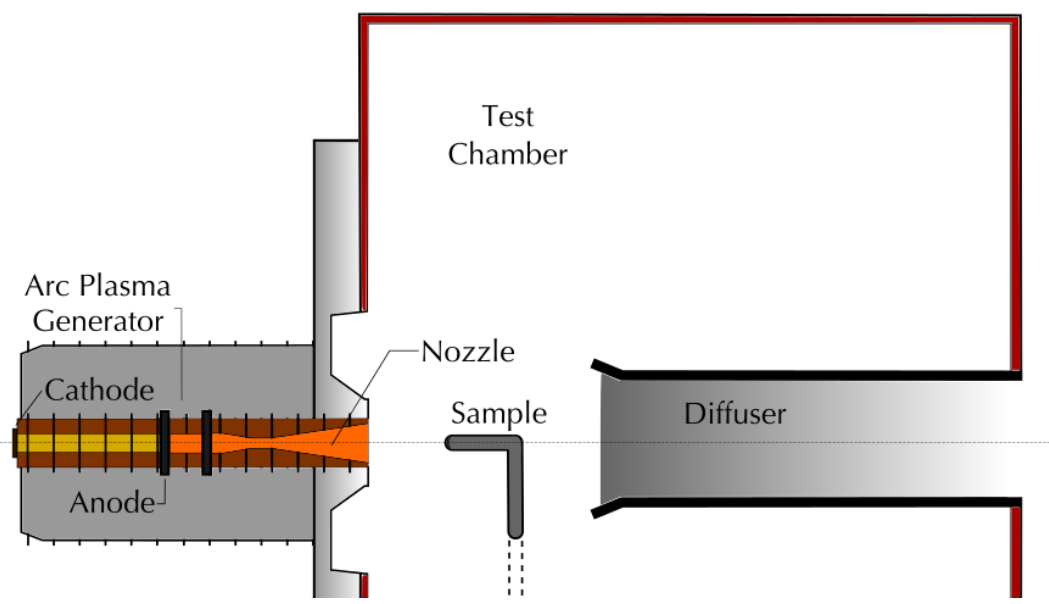

Figure 3: Schematic view of HyMETS test section. The plasma flow from the arc plasma generator is accelerated through the nozzle and exhausted into a vacuum test chamber where it stagnates on one of four water-cooled specimen/instrumentation injection stings arranged symmetrically around the inside circumference of the test chamber. Then, the flow is captured by a collector cone, followed by a constant diameter diffuser.

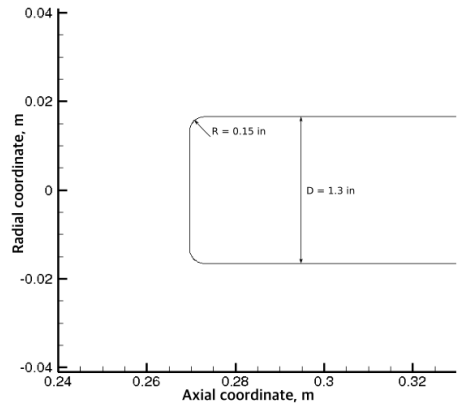

(a) Probe geometry.

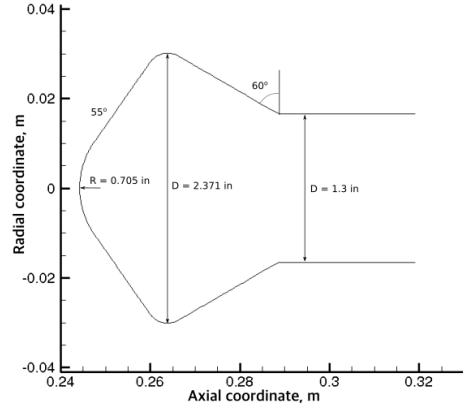

(b) Baby-SPRITE initial geometry.

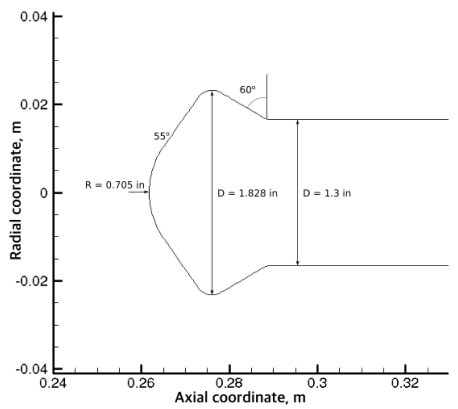

(c) Baby-SPRITE final geometry.

Figure 4: Probe (a) and baby-SPRITE (b, c) geometries tested in the 2.5-inch nozzle of the $400 \mathrm{~kW}$ HyMETS facility. The apexes of the models are located $2 \mathrm{in}, 1.0$ in and 1.7 in downstream from the nozzle exit plane $(\mathrm{NEP})$, respectively.

shear), only the outer mold line of the test specimen is needed. The time-dependent distribution of heat through the surface and the material recession can be calculated by a material thermal response code such as PATO ${ }^{6}$ with the CFD prediction of the environment as boundary conditions.

\section{Grid Generation}

The mesh generated for simulating the HyMETS environment is a 2D-axisymmetric multi-block structured grid, with six blocks. The computational grid was built with a loosely-coupled code using NASA's Chimera Grid Tools $(\mathrm{CGT})^{7}$ overset grid generation software and Pointwise grid generation software.

The grid generation process for the HyMETS environment simulations was performed as follows:

1. The geometries of the nozzle, the test chamber, the probe, and the baby-SPRITE were represented with curves.

2. The domain was then split into six blocks with the topology illustrated in Figure 5a for the probe, and in Figure 5b for the final baby-SPRITE. With this topology, the skewness equiangle of the cells is very small. The skewness can be examined with Pointwise after generating the blocks, and therefore the topology was changed several times until a overall minimum skewness was obtained. 


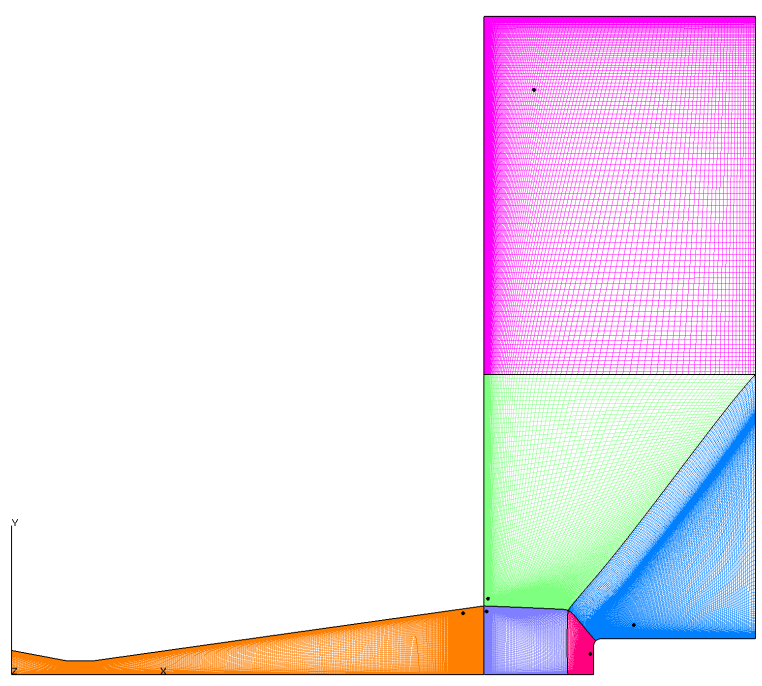

(a) Probe structured grids.

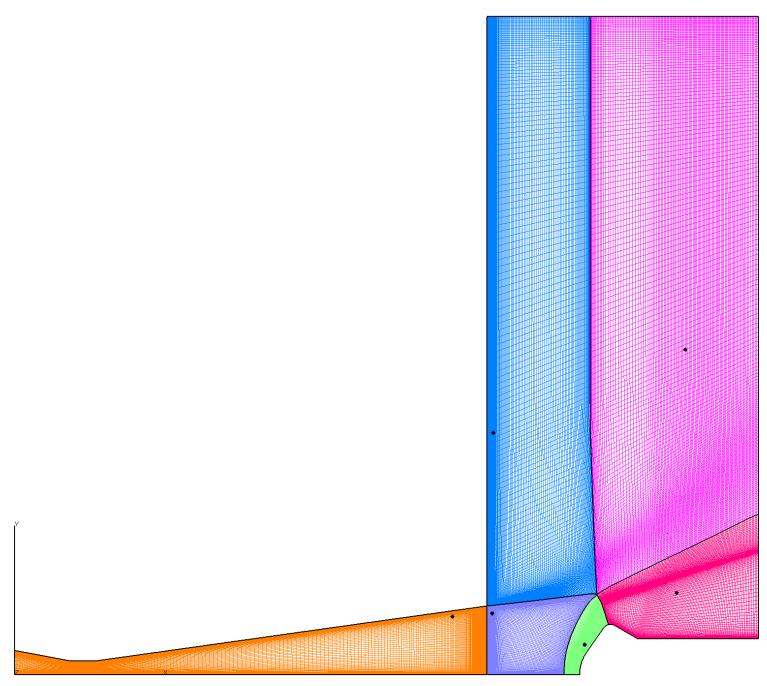

(b) Baby-SPRITE structured grids.

Figure 5: Multi-block structured mesh for the probe (a), and the final baby-SPRITE geometry (b) for HyMETS computions. There are six blocks, the grids are clustered near the walls and the shock. Shockalignment is an iterative process, manually obtained after running DPLR and regenerating the grids multiple times.

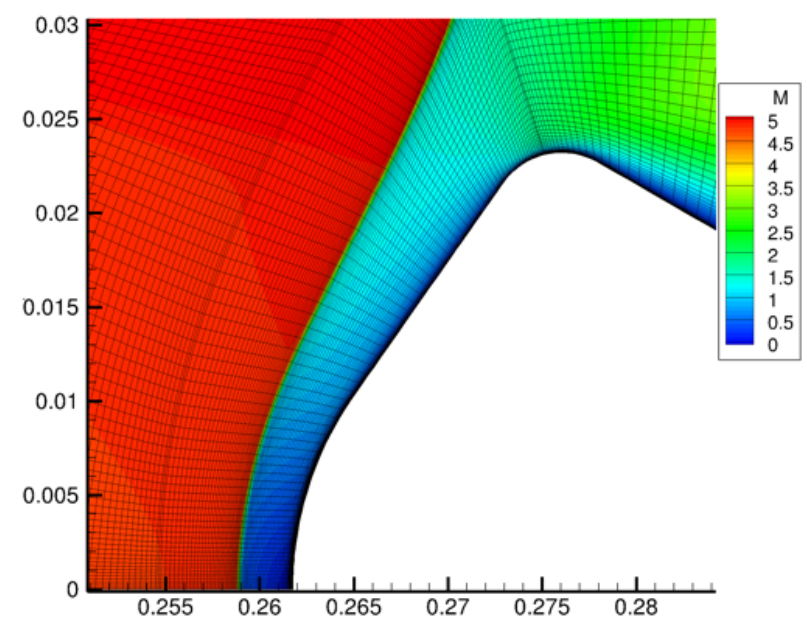

Figure 6: A close-up view of the baby-SPRITE final mesh simulated in a HyMETS environment. The mesh alignment with the shock is a semi-automatic process. This process is repeated until the shock is accurately captured.

3. Using CGT, structured surface grids are generated using elliptic schemes from the boundary curves that make up the topology. It is very important to ensure that there is continuity of grid points and spacings between blocks.

4. Small spacings and orthogonal grids are imposed near the walls and at the symmetry axis. The grids are coarsened from the walls in order to have a reasonable number of total cells in the computational domain. The maximum stretching ratio is controlled with the global variable $s r_{\max }=1.25$. 
5. Then, with a Glyph script, a Pointwise solver is applied in order to smooth the grids and reapply orthogonality at the walls and axis. After calling the solver, the connectivity between blocks is greatly improved thanks to smoother transitions.

6. Finally, CGT is called again to assemble the final mesh, as seen in Figure 5, and it is saved in Plot3D ASCII format.

The final refined grids for the probe and baby-SPRITE are shown in Figure 5. For the initial computational meshes, the grids in the blocks adjacent to the test specimen are not refined because the position of the shock is not known yet. After a converged solution is obtained for the coarse mesh, the shock in front of the probe or sample can be traced. The mesh generation script refines and aligns the grid on these blocks after manually defining the line where the shock is located. The hypersonic CFD code is then run with the refined mesh, and the shock location will slightly change compared to the previous solution. This process is repeated until the shock is accurately captured. Figure 6 shows a close-up view of the mesh and the Mach number contours for the final mesh. The grids accurately capture the shock everywhere.

\section{Numerical Methods}

All flow computations have been performed using DPLR, a parallel, structured-grid, finite-volume, Navier-Stokes code developed by Wright et al. ${ }^{8}$ For problems in which thermochemical nonequilibrium effects are important, the governing equations have been extended to account for finite-rate chemistry with multiple temperatures. The use of multi-block or overset grids allows for accurate resolution of complex geometries and flow structures. In the present modeling of an arc-jet test chamber, flows inside the nozzle and test chamber are assumed to be axisymmetric and steady. In order to provide the data at the boundary layer edge as an input for a material response code to estimate the surface recession due to ablation, the BLAYER $^{9}$ code has been employed.

The Navier-Stokes equations are a system of nonlinear equations. Since it is prohibitively expensive to solve the coupled nonlinear equations, the standard approach is to linearize the equations by approximating the convective flux vectors and dropping the high-order terms. The resulting equation for an unfactored implicit scheme still produces a large block-banded matrix that is very costly to invert and has very large memory requirements. Iterative relaxation schemes such as the Gauss-Seidel method have been used for more than a hundred years for solving a system of linear equations. The successive overrelaxation method reverts to the Gauss-Seidel method when the relaxation factor is set to 1.

Complex physical processes experienced by a vehicle flying at hypersonic speeds and a wide range of spatial and time scales make accurate and efficient computational modeling and simulation extremely challenging. Numerical stability problems arise from the stiffness of chemical source terms in the species concentration equations, whereas the need for numerical efficiency results from the large number of species equations that have to be solved along with the flow equations and the close coupling between the flow and chemistry.

An implicit scheme based on the symmetric point Gauss-Seidel method was developed to solve the NavierStokes equations and species transport equations in a fully coupled manner. ${ }^{10-12}$ It was also shown that the symmetric point Gauss-Seidel method was equivalent to an LU factorization, and hence the scheme was called LU-SGS. Diagonal dominance of a matrix is important for the convergence of the Gauss-Seidel method. Because limited computing power three decades ago precluded the inversions of large matrices, an approximate form of Jacobian matrices of the flux vectors was constructed using spectral radii to yield diagonal dominance. Despite being implicit, the resulting scheme requires only scalar diagonal inversions for nonreacting flows and block diagonal inversions for chemically reacting flows. Thus, computing time per iteration is usually less than some slowly-converging explicit schemes.

\section{Flux-splitting for Space}

The Navier-Stokes equations for a mixture of species in conservation law form can be written as: ${ }^{13}$

$$
\frac{\partial U}{\partial t}+\nabla \cdot\left(F-F_{v}\right)=W
$$

where $U$ is the vector of conserved variables and $W$ is the source term. Inviscid and viscous flux vectors are denoted by $F$ and $F_{v}$ respectively. Consider the upwind evaluation of the inviscid flux vector $F$ at a grid 
interface. The forward and backward moving fluxes, $F^{+}$and $F^{-}$, can be obtained by the Steger-Warming ${ }^{14}$ flux vector splitting approach:

$$
F=F^{+}+F^{-}=R^{-1} \Lambda^{+} R U+R^{-1} \Lambda^{-} R U
$$

where $R$ is the right eigenvector matrix and $\Lambda$ is the diagonal matrix of eigenvalues of the matrix $\partial F / \partial U$. Applying the flux vector splitting at a cell face gives:

$$
F_{i+1 / 2}=\left(R^{-1} \Lambda^{+} R\right)_{i} U_{i}+\left(R^{-1} \Lambda^{-} R\right)_{i+1} U_{i+1}
$$

Since this original Steger-Warming formulation is known to be highly dissipative, Candler and MacCormack $^{15}$ improved the method by evaluating the left and right eigenvalues and eigenvectors at the same location.

$$
F_{i+1 / 2}=\left(R^{-1} \Lambda^{+} R\right)_{i+1 / 2} U_{i}+\left(R^{-1} \Lambda^{-} R\right)_{i+1 / 2} U_{i+1}
$$

The flux can be written in the Roe flux form since $\Lambda^{ \pm}=(\Lambda \pm|\Lambda|) / 2$.

$$
F_{i+1 / 2}=\frac{1}{2}\left(R^{-1} \Lambda R\right)_{i+1 / 2}\left(U_{i}+U_{i+1}\right)+\frac{1}{2}\left(R^{-1}|\Lambda| R\right)_{i+1 / 2}\left(U_{i}-U_{i+1}\right)
$$

While the Roe averaging can be used to obtain the Roe scheme, a simple average of the primitive variables is used in practice.

To capture strong shock waves, each primitive variable in the eigenmatrices is averaged using a pressuredependent weight to revert smoothly to the original Steger-Warming flux. This weight is given by Eqs. 6a and $6 \mathrm{~b}$ :

$$
w_{i+1 / 2}=1-\frac{1}{2}\left(\frac{1}{(5 \delta p)^{2}+1}\right)
$$

where:

$$
\delta p=\frac{p_{i+1}-p_{i}}{\min \left(p_{i}, p_{i+1}\right)}
$$

The weight is applied so that the Roe flux is obtained in uniform pressure regions, and the original Steger-Warming flux is obtained as the pressure difference across the face becomes very large. High-order fluxes are obtained using the MUSCL approach with a weighted least-squares method to compute gradients.

\section{Gauss-Seidel Relaxation for Time}

The point Gauss-Seidel relaxation method can be written in two dimensions as:

$$
\begin{aligned}
\left(I+A_{i+1 / 2, j}^{+}-A_{i-1 / 2, j}^{-}+B_{i, j+1 / 2}^{+}-B_{i, j-1 / 2}^{-}\right) \delta U_{i, j}=H_{i, j} & +A_{i-1 / 2, j}^{+} \delta U_{i-1, j}-A_{i+1 / 2, j}^{-} \delta U_{i+1, j} \\
& +B_{i, j-1 / 2}^{+} \delta U_{i, j-1}-B_{i, j+1 / 2}^{-} \delta U_{i, j+1}
\end{aligned}
$$

where $A, B$ and $H$ denote the Jacobian matrices of flux vectors and the residual on the right-hand side. The equation can be solved in two steps.

Although the LU-SGS (or point Gauss-Seidel) scheme is parallelizable on shared memory computer systems, it cannot be parallelized easily on distributed-memory computer architectures. Candler et al. ${ }^{16}$ improved the parallelization of the LU-SGS scheme by replacing the forward and backward sweeps of the symmetric relaxation methods with a series of point Jacobi-like sub-iterations. Wissink et al. ${ }^{17}$ proposed a hybrid method that combined the Jacobi iterations for inter-processor communications and the LU-SGS for on-processor communications.

Jacobian matrices of the flux vectors should be diagonally dominant to ensure convergence to a steady state. However, the LU-SGS scheme using spectral radii for approximate Jacobian matrices suffered slow terminal convergence on highly stretched grids with high cell aspect ratios. Using exact plus and minus Jacobian matrices using similarity transformation matrices of the eigenvectors should improve convergence 
at the expense of an increased operation count to invert the full matrices. ${ }^{18}$ Wright et al. ${ }^{19}$ demonstrated that the full matrix form improved the convergence rates significantly on high cell aspect ratio grids to resolve the boundary layers of high Reynolds number flows.

Further, Wright et al. ${ }^{8}$ showed that the line Gauss-Seidel method enhanced the convergence rates over point relaxation in general, when the grid is highly stretched to resolve the boundary layer near the body surface in particular. That is, line relaxation can accelerate convergence by more strongly coupling the solution along the line of cells normal to the wall. The line Gauss-Seidel relaxation method can be written in two-dimensions as:

$$
\begin{array}{r}
B_{i, j+1 / 2}^{-} \delta U_{i, j+1}+\left(I+A_{i+1 / 2, j}^{+}-A_{i-1 / 2, j}^{-}+B_{i, j+1 / 2}^{+}-B_{i, j-1 / 2}^{-}\right) \delta U_{i, j}-B_{i, j-1 / 2}^{+} \delta U_{i, j-1} \\
=H_{i, j}-A_{i+1 / 2, j}^{-} \delta U_{i+1, j}+A_{i-1 / 2, j}^{+} \delta U_{i-1, j}
\end{array}
$$

The line Gauss-Seidel relaxation method can be parallelized by replacing the forward and backward sweeps with a series of line relaxations. The data-parallel line relaxation (DPLR) scheme can be written as:

$$
\begin{aligned}
\left(\tilde{A^{n}}-\tilde{C^{n}}\right) \delta U^{k} & =H^{n}-\tilde{C^{n}} \delta U^{k-1} \\
\delta U^{n+1} & =\delta U^{k_{\max }}
\end{aligned}
$$

where $\tilde{C^{n}}$ comprises all the off-diagonal blocks of the implicit operator $\tilde{A^{n}}$. This equation must be iterated several $\left(k_{\max }\right)$ times, as indicated by the superscript $k$, until the solution converges.

\section{Chemical Reaction Model}

$\operatorname{Park}^{20}$ developed chemical reaction models for finite-rate thermochemical nonequilibrium phenomena. For each cell, the global density $\rho$, velocity components $u$ and $v$, energy per unit volume $e$, the sum of vibrational and electronic energy $e_{v}+e_{e}$, and number densities $n_{i}$, are available. Using these quantities, the translationalrotational temperature can be calculated by:

$$
e=\left(\sum_{i} C_{v i} n_{i}\right) T+e_{v}+e_{e}+\sum_{i} h_{0 i} n_{i}+\frac{\rho}{2}\left(u^{2}+v^{2}\right)
$$

In the two-temperature model, the rate coefficients are assumed to be functions of the average temperature $T_{a}$, which is a geometrically averaged temperature between $T$ and $T_{v}$, defined as:

$$
T_{a}=\sqrt{T_{v} T}
$$

The air model used here is Park's 6-species model, comprising $\mathrm{N}_{2}, \mathrm{O}_{2}, \mathrm{NO}, \mathrm{N}, \mathrm{O}$, and Ar, with 8 reactions:

$$
\begin{aligned}
& \mathrm{N}_{2}+\mathrm{M} \rightleftharpoons \mathrm{N}+\mathrm{N}+\mathrm{M} \\
& \mathrm{O}_{2}+\mathrm{M} \rightleftharpoons \mathrm{O}+\mathrm{O}+\mathrm{M} \\
& \mathrm{NO}+\mathrm{M} \rightleftharpoons \mathrm{N}+\mathrm{O}+\mathrm{M} \\
& \mathrm{N}_{2}+\mathrm{Ar} \rightleftharpoons \mathrm{N}+\mathrm{N}+\mathrm{Ar} \\
& \mathrm{O}_{2}+\mathrm{Ar} \rightleftharpoons \mathrm{O}+\mathrm{O}+\mathrm{Ar} \\
& \mathrm{NO}+\mathrm{Ar} \rightleftharpoons \mathrm{N}+\mathrm{O}+\mathrm{Ar} \\
& \mathrm{N}_{2}+\mathrm{O} \rightleftharpoons \mathrm{NO}+\mathrm{N} \\
& \mathrm{NO}+\mathrm{O} \rightleftharpoons \mathrm{O}_{2}+\mathrm{N} \\
& \hline
\end{aligned}
$$

Table 1: Park's six species with eight reactions chemistry model. 


\section{BLAYER}

The boundary layer at the surface of an atmospheric entry vehicle is the region where the enthalpy of the free stream flow is being dissipated by viscosity, transferring energy in the form of heat. Real-gas Navier-Stokes flow solvers routinely model this phenomenon as part of predicting the aerothermodynamic environments that entry vehicles must be designed to withstand. The importance of knowing boundary layer properties and their relation to possible transition from laminar to turbulent flow is illustrated by Space Shuttle Discovery mission STS-114, when the appearance of tile gap filler protrusions on the underside of the nose was deemed serious enough that a crew member was called upon to exit the vehicle and remove the potential threat prior to descent from orbit.

BLAYER $^{9}$ was developed in support of the DPLR flow solver. The essence of the algorithm is to locate the boundary layer edge by seeking the peak curvature in a total enthalpy profile. Turning that insight into a practical tool suited to a wide range of possible profiles has led to a hybrid two-stage method. BLAYER reads a volume dataset extracted from a flow solution and writes a surface dataset containing three sets of values: wall data, boundary layer edge-related data, and values either at a specified surface roughness height or at the estimated momentum thickness height. Boundary layer edge locations can be affected by the particular choice of boundary layer profile and the associated normalizations. There are at least two choices of total enthalpy profile, and these can produce different calculated edge locations. Derived quantities such as displacement thickness and momentum thickness, on the other hand, should be insensitive to the profile choice. BLAYER handles 3-D and 2-D/axisymmetric flows and a variable number of gas species for any atmosphere. It allows for more than one temperature and for optional extra flow quantities.

The traditional approach of defining the boundary layer edge at a given body point as the location nearest to the body of $99.5 \%$ of the free-stream total enthalpy normally suffices for the two-dimensional flows upon which that choice is based. Three-dimensional flow can present boundary layer profiles that may differ significantly from their two-dimensional counterparts by undershooting or overshooting the freestream reference value in the region of interest. The total enthalpy ratio does not necessarily asymptote to one near the body. Indeed, enough of an undershoot can mean that the traditional method fails completely. BLAYER's hybrid method uses a curvature-based scheme to locate the likely neighborhood of the edge in the total enthalpy ratio profile, then apply the $99.5 \%$ rule to the peak ratio in that neighborhood. For well-behaved profiles, this is consistent with the traditional edge method. In the presence of overshoots or undershoots, the hybrid method inevitably produces edge thicknesses differing from traditional method results, but plausible results for irregular profiles can be obtained when the traditional method fails.

\section{Results}

Axisymmetric thermochemical non-equilibrium hypersonic flow simulations have been carried out using the DPLR code for both the probe and the baby-SPRITE capsule models, with finite-rate chemistry and Park's two-temperature model. Radiative equilibrium and cold wall boundary conditions are imposed at the surface of the probe and the capsule. Grids are generated using CGT and Pointwise.

While the non-catalytic condition is applied to the nozzle and chamber wall, the radiative equilibrium and catalytic boundary condition is used for the probe or capsule body with a catalytic efficiency of 1 and an emissivity of 0.85 . A cold wall at $350 \mathrm{~K}$ is specified at the surface. At the inlet, for simplicity, a uniform flow condition is specified at $3832 \mathrm{~K}, 0.1125 \mathrm{~kg} / \mathrm{m}^{3}$, and $0.0208 \mathrm{~km} / \mathrm{s}$. The outlet chamber pressure is 0.0228 $\mathrm{kPa}$. For numerical stability, flow inside the chamber is initialized with a low density.

First, the probe used in the facility to calibrate the test conditions has been simulated. The effects of shock-grid alignment and grid spacing at the walls were analyzed. Then, the initial baby-SPRITE geometry with a base diameter of $\mathrm{D}=2.371$ in was simulated. Flow blockage and unsteady flow oscillations were observed in this simulation. The size of the baby-SPRITE capsule was reduced until finding that a diameter of $\mathrm{D}=1.828$ in avoids flow blockage and reaches the expansion desired. Detailed flow computations are performed for the final baby-SPRITE geometry.

The resulting heat flux and pressure have been calculated for the probe and the final baby-SPRITE geometry. With the heat flux and pressure, the material recession can be predicted with a material thermal response code such as PATO. 


\section{A. Probe Simulations}

The computational results for the calorimetric probe with the dimensions shown in Figure 4a are presented in this section.

First, the effect of wall spacing is analyzed. Previous computational work ${ }^{3,4}$ with HyMETS used a wall spacing of $\Delta s_{\text {wall }}=10^{-5} \mathrm{~m}$. In this work, we have compared the effect of the wall spacing by simulating three different cases:

- $\Delta s_{\text {wall }}=10^{-5} \mathrm{~m}$ for all walls

- $\Delta s_{\text {wall }}=10^{-5} \mathrm{~m}$ for the chamber walls, and $\Delta s_{\text {wall }}=10^{-6} \mathrm{~m}$ for the nozzle and probe walls

- $\Delta s_{\text {wall }}=10^{-6} \mathrm{~m}$ for all walls

Figure 7 shows the Mach number contours for these three cases. The fourth image shows a photograph taken of the probe inside HyMETS. The grids with coarser resolution on walls fail to capture the correct plume boundary. Therefore, all grids in this study have wall spacing of $\Delta s_{\text {wall }}=10^{-6} \mathrm{~m}$.

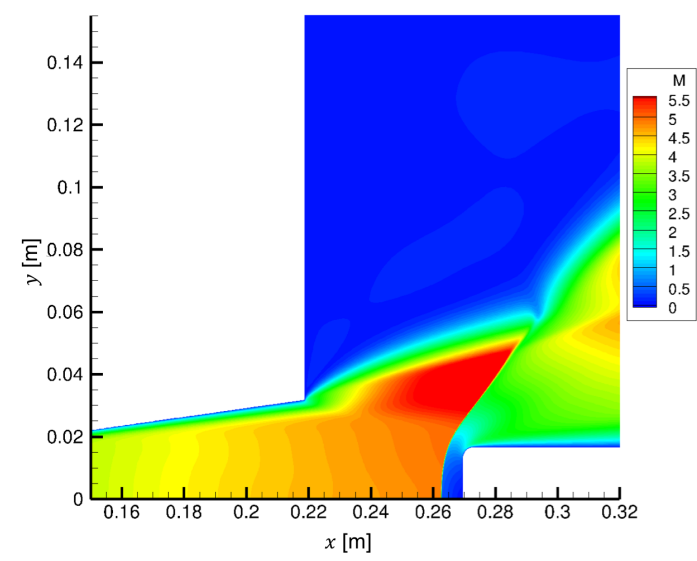

(a) $\Delta s_{\text {wall }}=10^{-5} \mathrm{~m}$ everywhere.

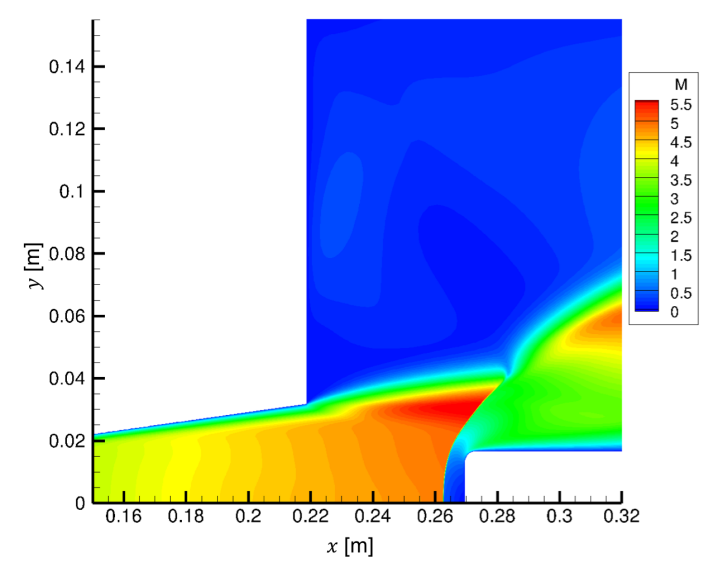

(c) $\Delta s_{\text {wall }}=10^{-6} \mathrm{~m}$ everywhere.

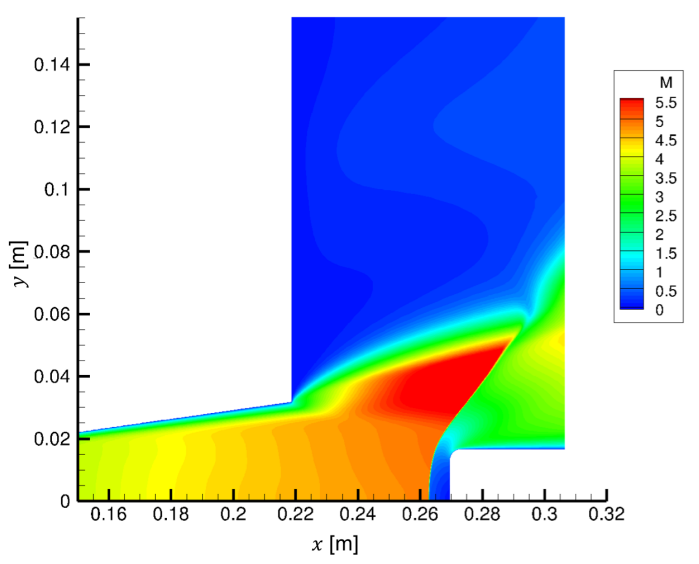

(b) $\Delta s_{\text {wall }}=10^{-5} \mathrm{~m}$ and $\Delta s_{\text {wall }}=10^{-6} \mathrm{~m}$.

Figure 7: Effect of wall grid spacing $\Delta s_{\text {wall }}$ on flow solution.

The next problem analyzed is the effect of shock-grid alignment for the probe. The process can be seen in Figure 8. A guess of the shock location was used for generating the initial grid. After running the CFD code, the placement of the shock is obtained. It can be observed in Figure 8a that the thickness of the shock is relatively big: there are only two to three cells in this region. The placement of the shock is updated using the grid generation code, and grids are then regenerated; see Figure $8 \mathrm{~b}$ for an intermediate grid solution. 
This process was repeated until the grids from Figure 8c were obtained. The final grids align with and capture the shock very well.

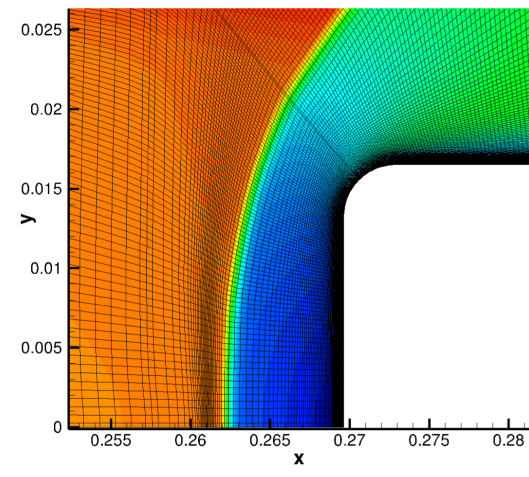

(a) Initial grid

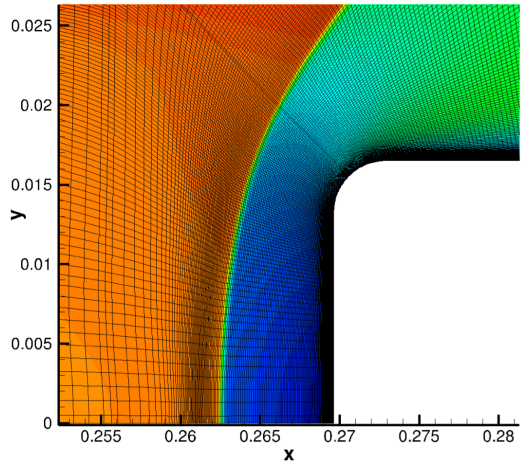

(b) Intermediate grid

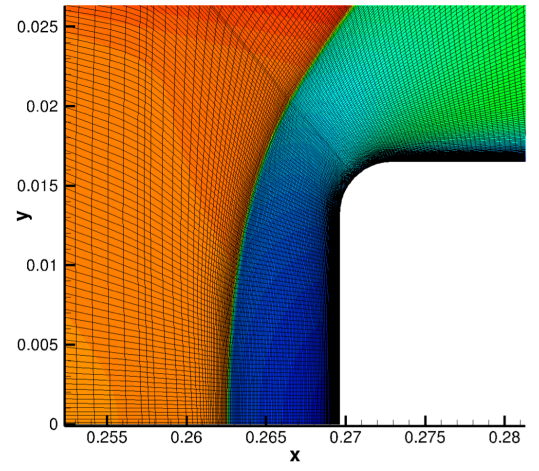

(c) Final grid

Figure 8: Effect of shock-grid alignment on flow solution for the probe.

The flow solution for the complete domain using the final grid is represented in Figure 9. The Mach number contours are shown. It is observed that the shock is accurately captured by aligning the grid to the shock. The test specimen wall has been simulated with radiative equilibrium and catalytic boundary conditions, with cold wall conditions $(350 \mathrm{~K})$. The nozzle and chamber walls are assumed to be cold (350 K) and non-catalytic.

Figure 10 shows the film coefficient $C_{H}\left(\mathrm{~kg} /\left(\mathrm{m}^{2} \mathrm{~s}\right)\right)$ and the pressure $p_{w}(\mathrm{kPa})$ distributions at the wall of the probe, calculated with BLAYER, for an intermediate DPLR solution and for the final grid-converged solution. The film coefficient is a quantity typically required by material response codes, and it is defined as:

$$
C_{H}=\frac{q_{w}}{H_{\text {edge }}-H_{\text {wall }}}
$$

where $q_{w}\left(\mathrm{~W} / \mathrm{m}^{2}\right)$ is the heat flux at the wall, $H_{\text {edge }}(\mathrm{J} / \mathrm{kg})$ is the enthalpy at the boundary layer edge, and $H_{\text {wall }}$ is the enthalpy at the wall.

The difference between the $C_{H}$ and $p_{w}$ distributions over the probe for the initial and final grids shown in Figure 8 is relatively small. The distributions differ the most when closer to the stagnation point. It is worth mentioning that the initial grid incorporated a very good first guess of the shock location. Thus, the heat flux and pressure distributions are very similar when comparing the initial and final grid solutions.

\section{B. Initial Baby-SPRITE Simulations}

In this section the computational results for the baby-SPRITE capsule with a base diameter of $D=2.371$ in are shown. The geometry details of this baby-SPRITE are shown in Figure 4b. The mesh is shown in Figure 11. This initial geometry for the baby-SPRITE appears to be too big and too close to the nozzle exit plane, leading to potential flow blockage, see Figure 12. There are unsteady shock-plume interactions, as it can be seen in the images. In addition, the flow is under-expanded and it cannot reach the designed Mach of $M=5$ at the stagnation line.

Figure 13 shows the Mach and pressure contours for the initial baby-SPRITE. It is clear that the flow is under-expanded, due to the close proximity of the capsule to the NEP.

The residuals are plotted in Figure 14. It can be observed that the residuals fluctuate in a somehow periodic manner, suggesting that the shock-plume interactions may be periodic. Simulations were run for many iteration steps to see if these fluctuations would disappear after some time, but they did not.

After obtaining the computational results for the flow, it was decided that the base diameter had to be reduced. It was desirable to keep the same shape of the SPRITE geometry, that is, a $55^{\circ}$ sphere-cone, with nose-radius of $0.705 \mathrm{in}$, and an aft shell with $30^{\circ}$ inclination. Consequently, the only variable that could be changed was the base diameter. However, there is a lower limit to the minimum base diameter: the 


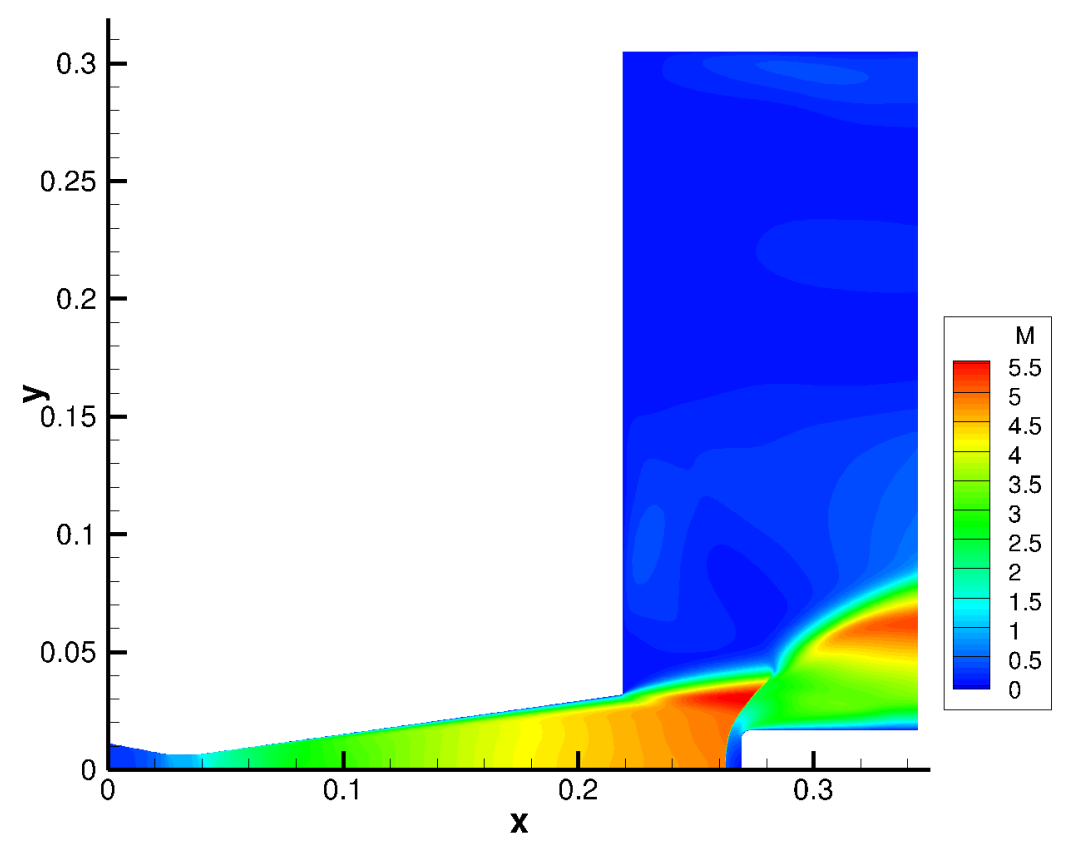

Figure 9: DPLR flow solution with the final grid for the probe. Mach number contours.

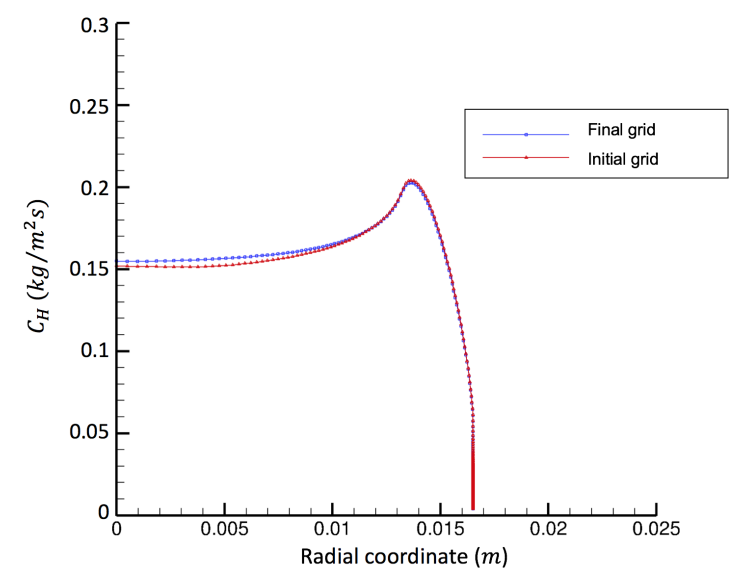

(a) Film coefficient distribution $C_{H}\left(\mathrm{~kg} /\left(\mathrm{m}^{2} \mathrm{~s}\right)\right)$

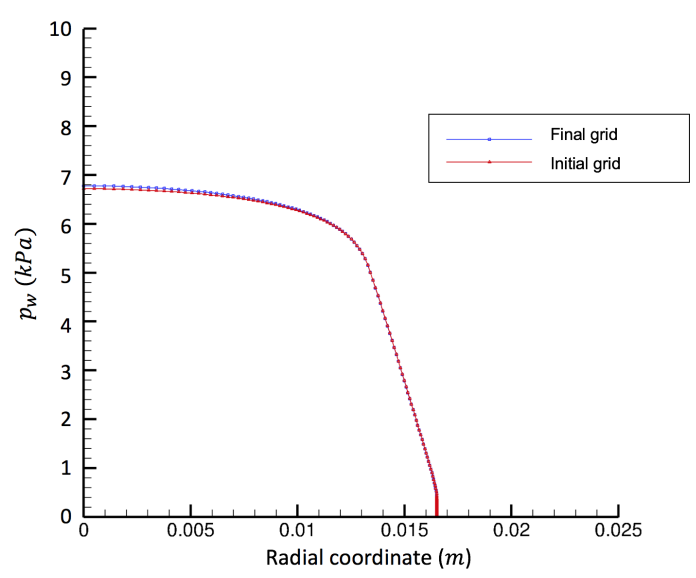

(b) Wall pressure $p_{w}(\mathrm{kPa})$

Figure 10: Effect of shock-grid alignment on heat flux and pressure distribution over the probe in HyMETS. The final grid solution is shown in blue, and the initial grid is in red. 


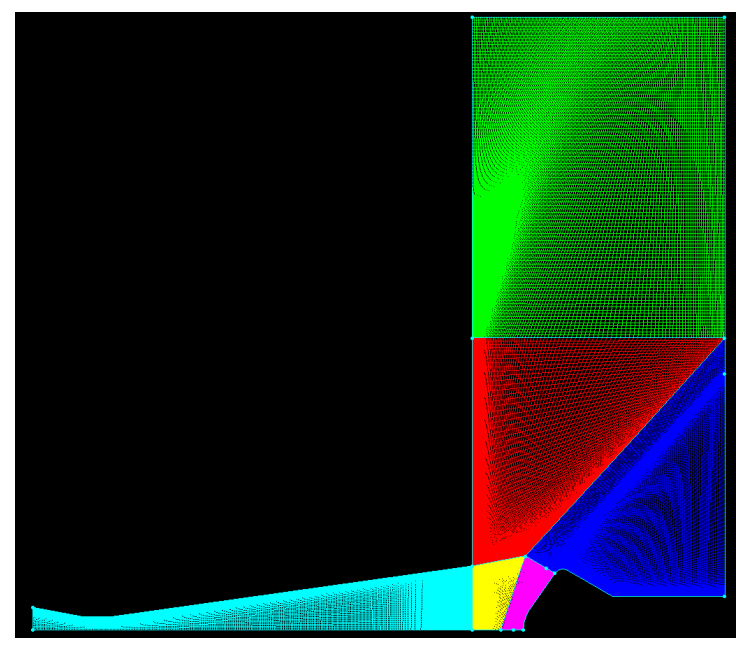

Figure 11: Computational grid for the initial capsule geometry, with a base diameter of 2.371 in.
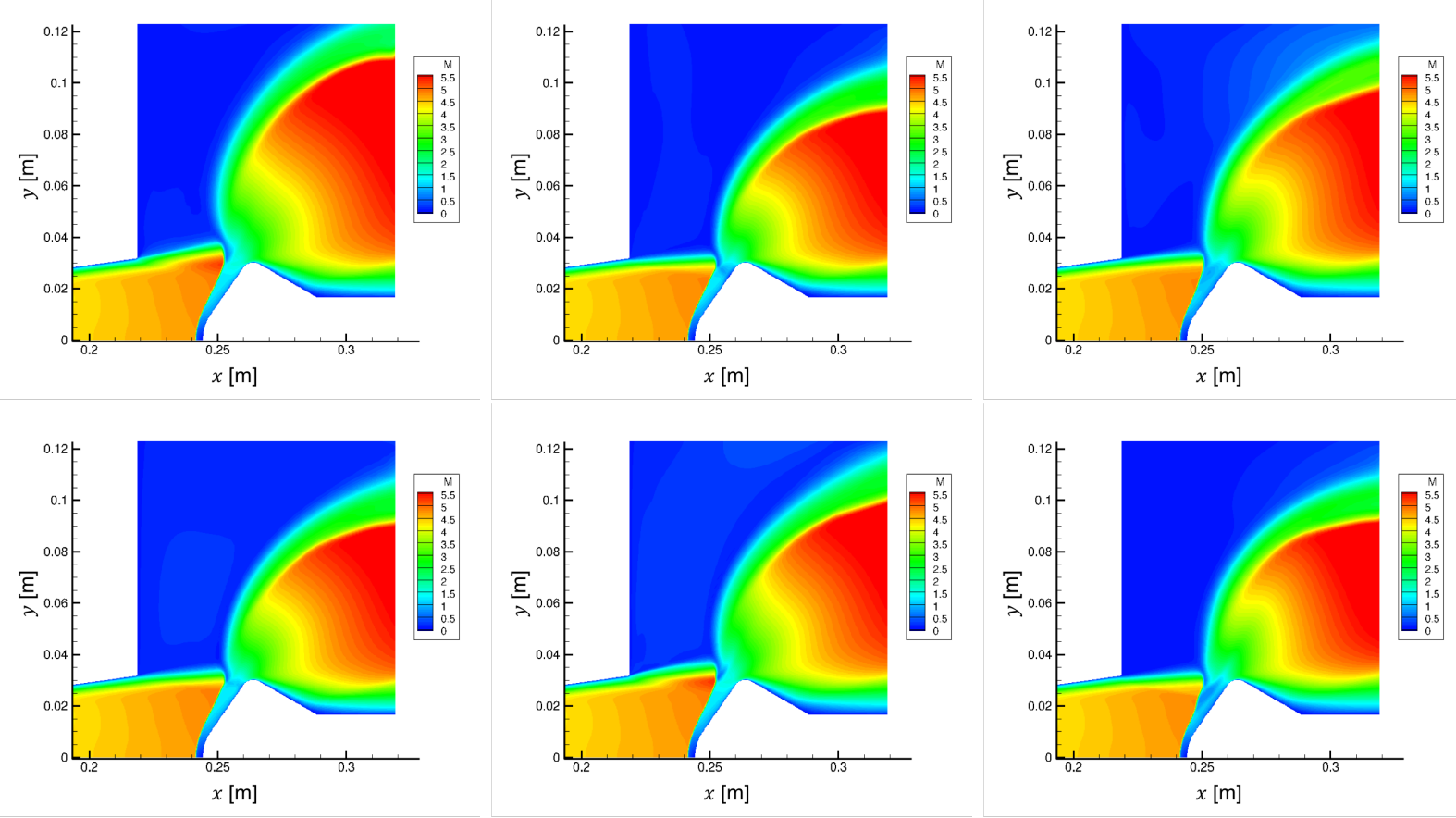

Figure 12: Mach number contours for the baby-SPRITE capsule with $D=2.371$ in, for different computational time steps. There are unsteady shock-plume interactions, and the flow is under-expanded and it cannot reach $M=5$ at the stagnation line. There might be supersonic flow overflowing the diffuser.

thermocouples have fixed dimensions, and a PICA outer shell that is too small will not fit the thermocouples. The baby-SPRITE design was initially changed to a base diameter of $D=2$ in.

\section{Intermediate Baby-SPRITE Simulations}

With a base diameter of $D=2$ in, new baby-SPRITE simulations were performed. The periodic behavior of the residuals is still present for this case, see Figure 15, but with better convergence.

The flow conditions have improved but still not enough. The flow is not expanded enough due to the proximity of the baby-SPRITE to the NEP; see Figure 16. The Mach number at the stagnation line reaches a higher value than the initial baby-SPRITE but still relatively far from $M=5$. Flow blockage may still be 


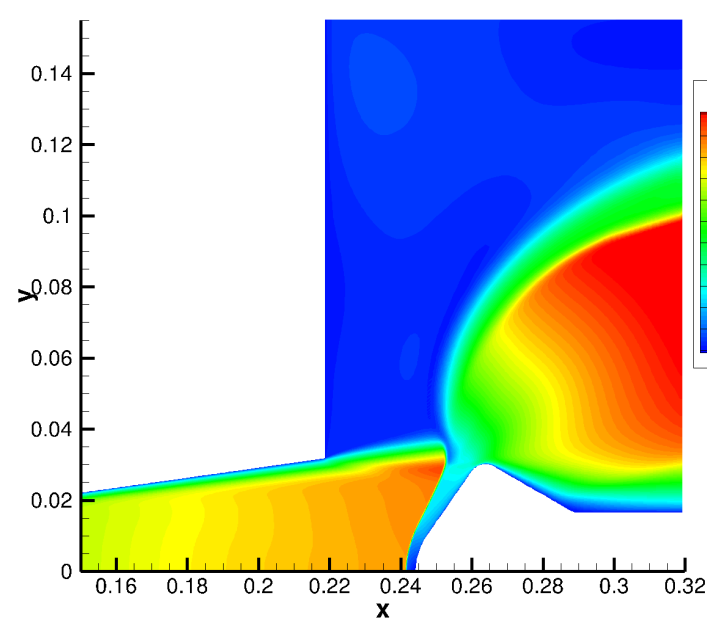

(a) Mach number contours

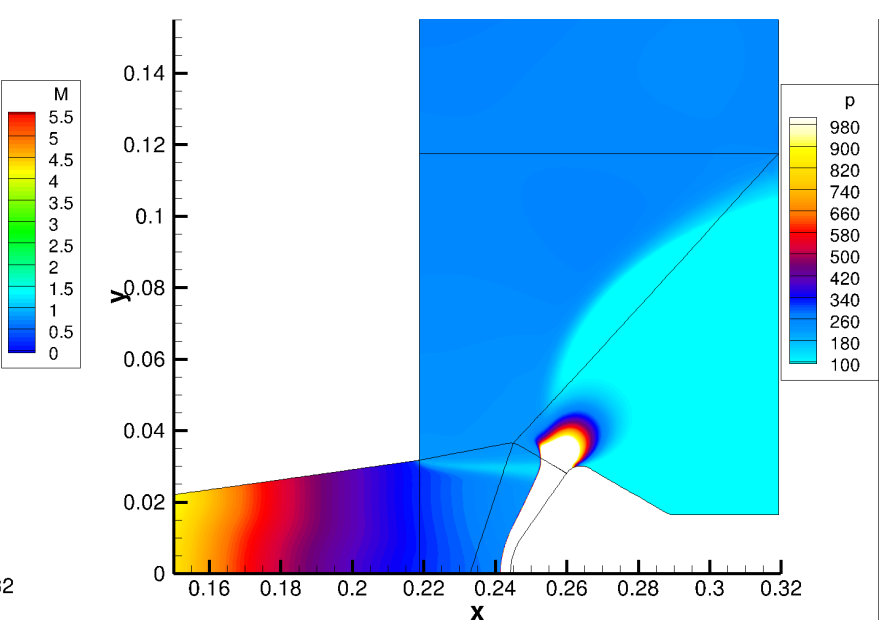

(b) Pressure contours ( $\mathrm{Pa})$

Figure 13: Flow solution for the initial baby-SPRITE, with $D=2.371$ in.

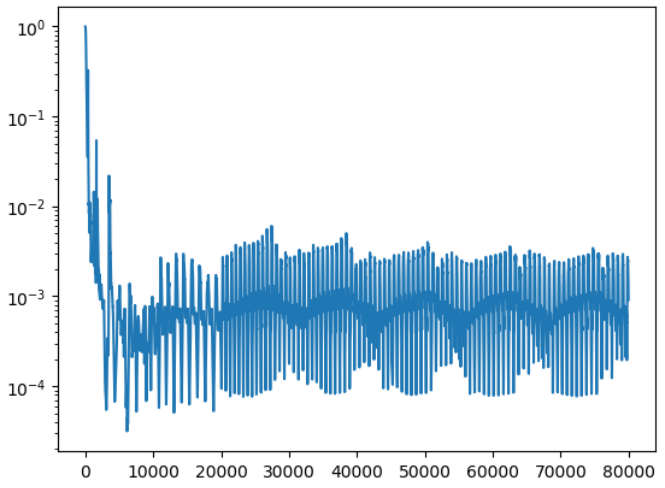

Figure 14: Residuals for the initial baby-SPRITE.

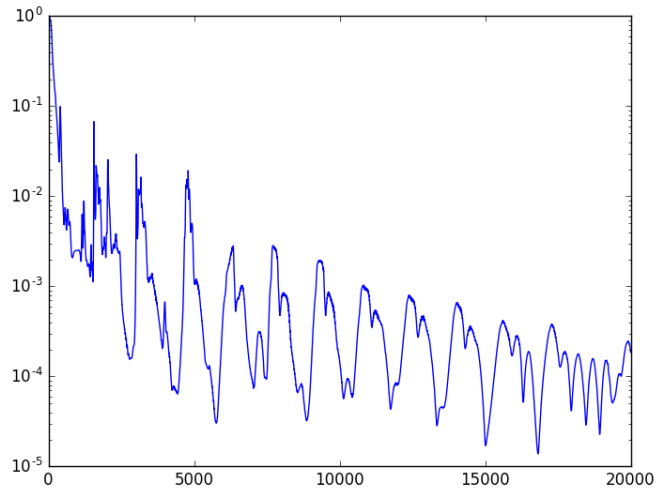

Figure 15: Residuals for the intermediate baby-SPRITE. 
an issue with this baby-SPRITE sizing.

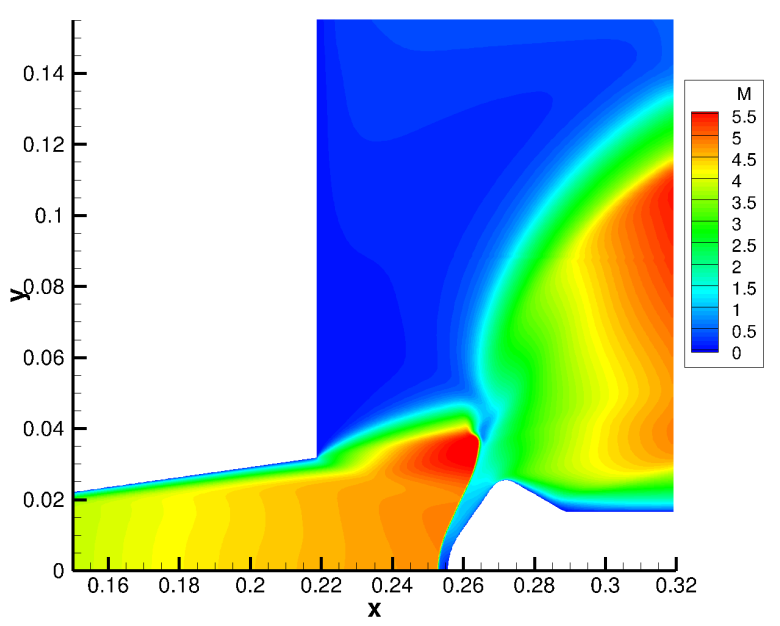

(a) Mach number contours

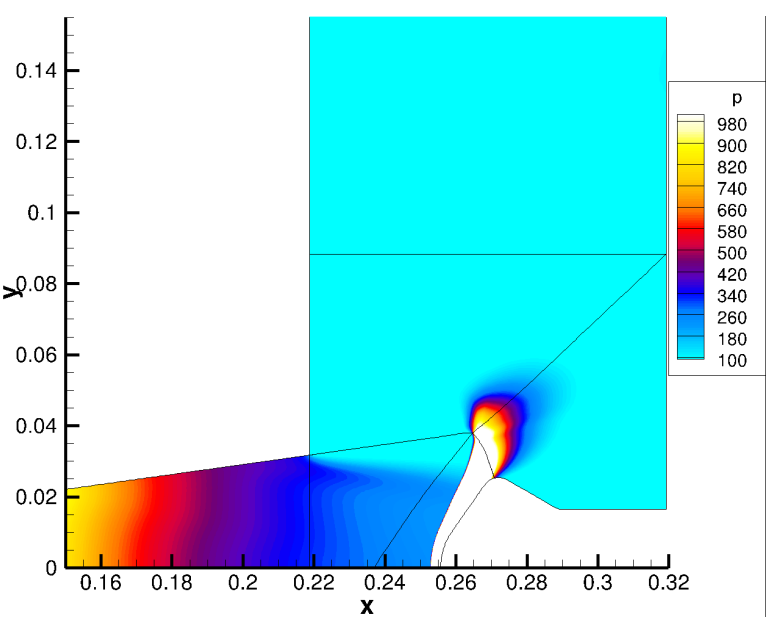

(b) Pressure contours $(\mathrm{Pa})$

Figure 16: Flow solution for the intermediate baby-SPRITE, with $D=2.0$ in.

The design of the baby-SPRITE had to be changed again. The base diameter was originally changed to $D=1.8 \mathrm{in}$, but finally it was decided to keep a base diameter of $D=1.828$ in, which would fit the thermocouples without problems. In this study, we will only show the $D=1.828$ in case, however the results were very similar due to the similar dimensions.

Table 2 summarizes the base diameters and the distances to the NEP, for the different specimens simulated in this work.

\begin{tabular}{|l|c|c|c|c|}
\hline Specimen & Probe & Baby-SPRITE \#1 & Baby-SPRITE \#2 & Baby-SPRITE \#3 \\
\hline Base diameter (in) & 1.3 & 2.371 & 2.0 & 1.828 \\
Distance to NEP (m) & 0.051 & 0.0251 & 0.0367 & 0.0428 \\
\hline
\end{tabular}

Table 2: Distance downstream from apex of each specimen to nozzle exit plane.

\section{Final Baby-SPRITE Simulations}

The results for the final baby-SPRITE geometry are shown in this section. The reduction to a base diameter $D=1.828$ in improved the flow conditions. Figure 17 shows the residuals. The periodic fluctuations have completely disappeared and fast convergence is reached.

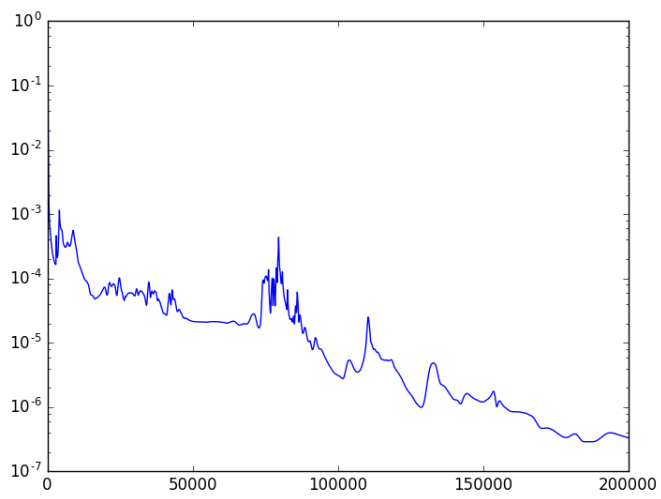

Figure 17: Residuals for the final baby-SPRITE. 
The flow solution (represented by the Mach and pressure contours) is shown in Figure 18. A Mach number close to 5, the desired one, is obtained at the stagnation line. In addition, it can be observed that the flow seems to be properly expanded, as represented on Figure 18b. Because we have reduced the base diameter, the distance to the NEP has increased, so the flow has enough space to expand to reach the required Mach number conditions.

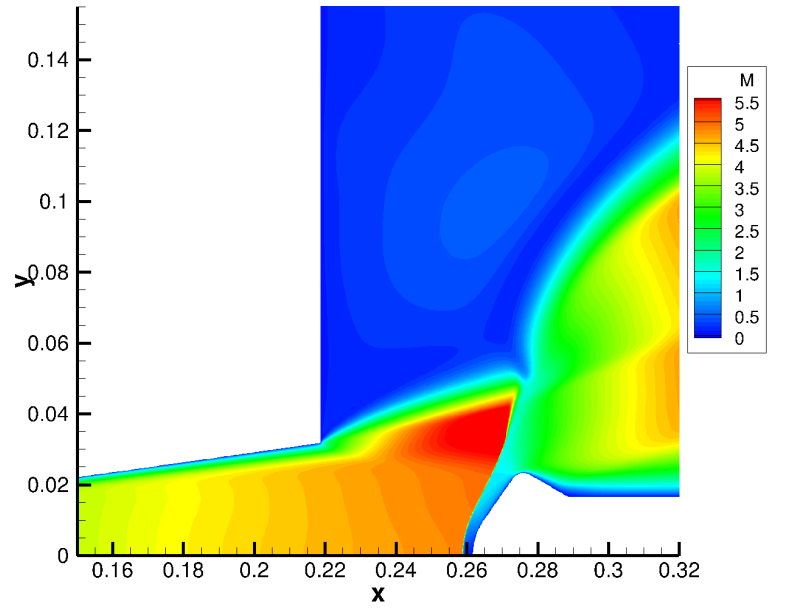

(a) Mach number contours.

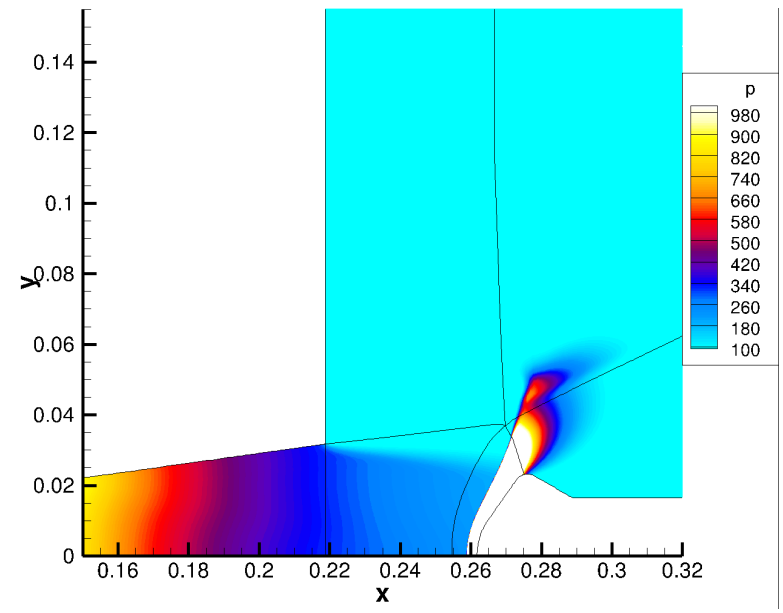

(b) Pressure contours $(\mathrm{Pa})$.

Figure 18: Flow solution for the final baby-SPRITE, with $D=1.828$ in.

The shock-grid alignment process for the final geometry is shown in Figure 19. The initial guess for the shock placement was not as good as with the probe, and more iterations were needed to find the final shock position; see Figure 19c for the final computational grid.

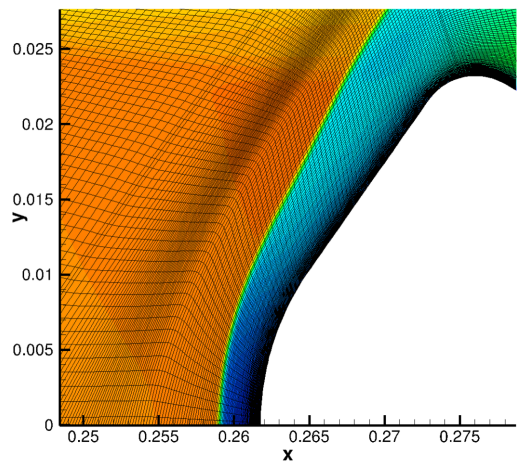

(a) Initial grid

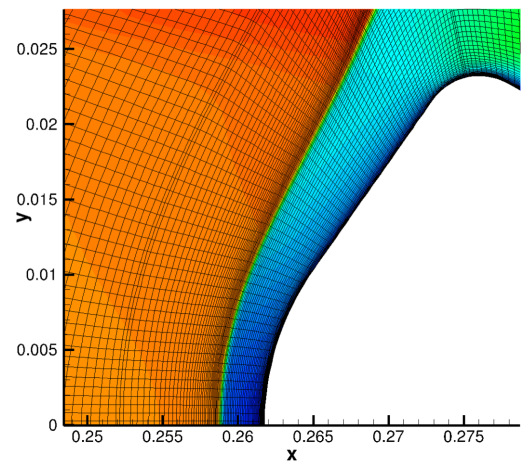

(b) Intermediate grid

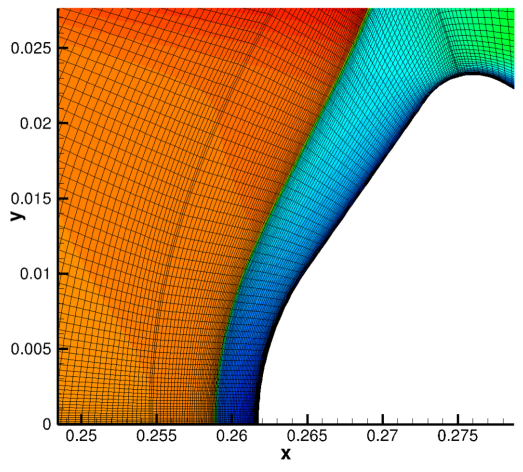

(c) Final grid

Figure 19: Effect of shock-grid alignment on flow solution for the baby-SPRITE with base diameter $D=$ $1.828 \mathrm{in.}$

The flow solution for the complete domain for the final grid is represented in Figure 20. The Mach number contours are shown. The shock is accurately captured by aligning the grid to the shock. The capsule wall has been simulated with radiative equilibrium and catalytic boundary conditions, with cold wall (350 $\mathrm{K})$. The nozzle and chamber walls are assumed to be cold $(350 \mathrm{~K})$ and non-catalytic.

To end this study, a comparison of the heat flux and wall pressure is presented. Figure 21 shows the heat flux and wall pressure for the capsule for the initial, intermediate and final grids. The initial grids were not orthogonal at the wall nor at the axis, and there was not grid alignment with the shock, as seen in Figure 19a. One of the main assumptions made in BLAYER to calculate the surface properties on hypersonic flows is that the radial grid lines are normal to the surface (at least in the boundary layer region). Consequently, the prediction of the $C_{H}$ and $p_{w}$ differ significantly between the initial and final grids. In addition, near the 


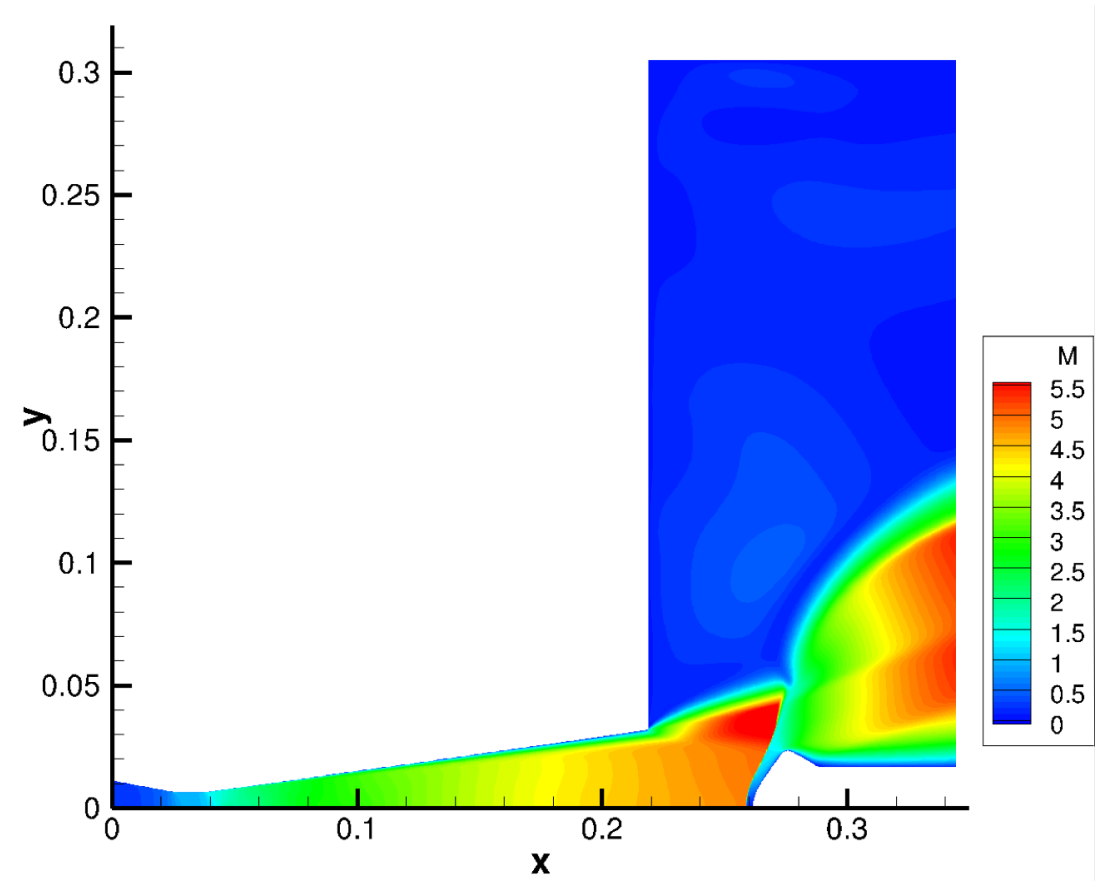

Figure 20: DPLR flow solution with the final grid for the baby-SPRITE with base diameter $D=1.828$ in. Mach number contours.

stagnation point, the $C_{H}$ slightly decreases and then increases. This is due to the non-orthogonality of the grids at the axis. For the intermediate grids, the distributions of both $C_{H}$ and $p_{w}$ seem to match with the final grid. However, there is a small kink at the stagnation point on the $C_{H}$ : this is due to the grids not being fine enough near the axis, see Figure 19b. The final grid was refined near the axis, and better aligned with the shock, obtaining the proper $C_{H}$ distribution near the stagnation point.

Figure 22 shows the film coefficient $C_{H}$ and the pressure $p_{w}$ distributions at the wall of the probe and the sample, for the shock-aligned final grids of each specimen, see Figures $8 \mathrm{c}$ and $19 \mathrm{c}$ for reference. The pressure near the stagnation point is very similar. However, the distributions of $C_{H}$ and $p_{w}$ have different shapes. The geometry of the test specimen changes significantly these distributions.

Hypersonic CFD simulations can improve testing capabilities and knowledge of the test article environment.

\section{Conclusions}

Pre-test high-fidelity computational simulations have been performed to design a capsule model for arcjet testing in support of Mars 2020 mission. Axisymmetric thermochemical non-equilibrium hypersonic flow simulations have been carried out using the DPLR code with finite-rate chemistry and Park's twotemperature model. Radiative equilibrium and cold wall boundary conditions are imposed at the capsule surface. Effects of grid quality on the accuracy of solutions have been investigated. Grid alignment with the bow shock, grid orthogonality at the axis of symmetry, and the fine grid clustering near the wall for both nozzle and chamber have been critical for accurate solutions whose quality is high enough for material response analysis. While grid alignment and orthogonality are essential for accurate assessment of surface heat flux using the BLAYER code, wall grid spacing has significant effect on prediction of the nozzle plume boundary. The interactions of the plume boundary and the bow shock wave appear to characterize the high-temperature reacting flow environment inside the arc-jet test chamber. A design study has determined the size of the capsule model called Baby-SPRITE to avoid flow blockage and hence possible damage to the experimental facility. Post-test simulations will be performed to calibrate and enhance the computational models. 


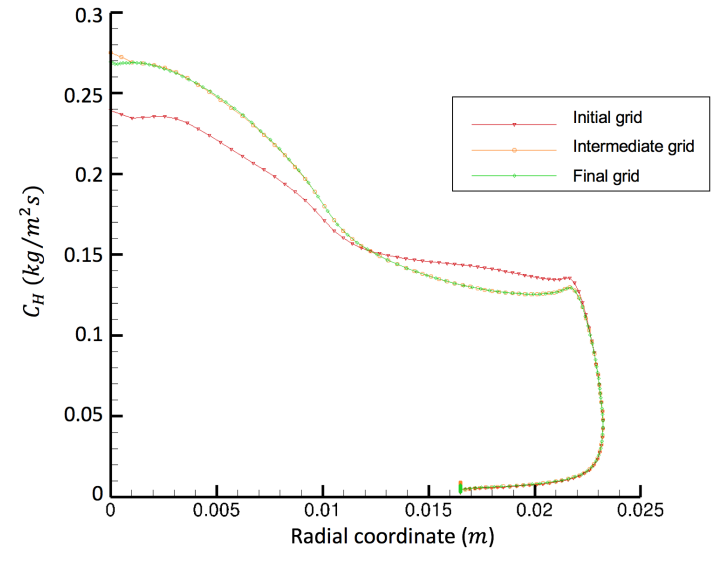

(a) Film coefficient distribution $C_{H}\left(\mathrm{~kg} /\left(\mathrm{m}^{2} \mathrm{~s}\right)\right)$

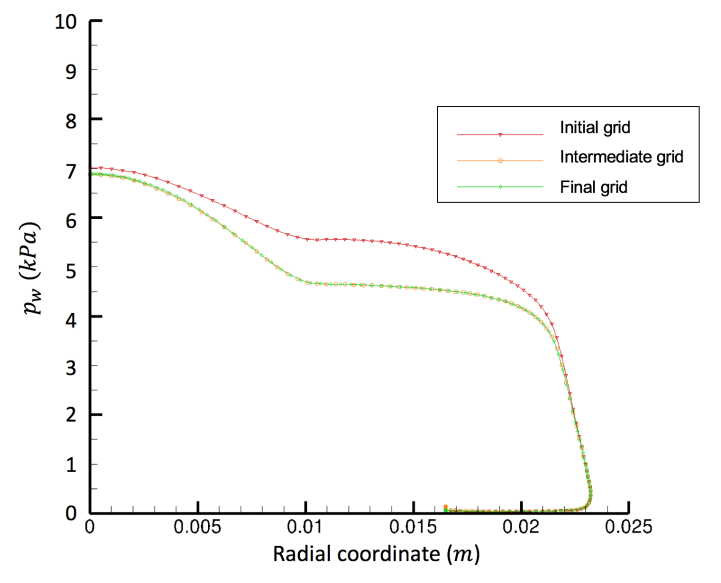

(b) Wall pressure $p_{w}(\mathrm{kPa})$

Figure 21: Heat flux and pressure distribution over the baby-SPRITE in HyMETS, for different computational grids. The initial grid BLAYER solution is shown in red, the intermediate grid is shown in orange, and the final grid is shown in green. Orthogonal, shock-aligned, refined grids are necessary to capture accurately the $C_{H}$ and $p_{w}$ distributions.

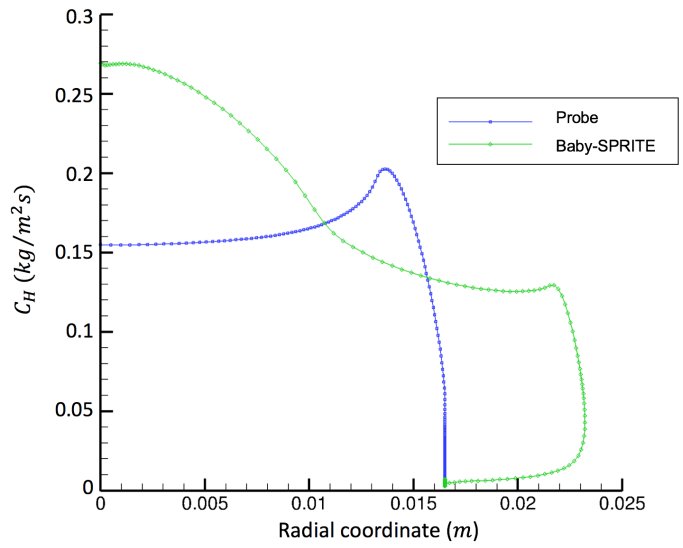

(a) Film coefficient distribution $C_{H}\left(\mathrm{~kg} /\left(\mathrm{m}^{2} \mathrm{~s}\right)\right)$

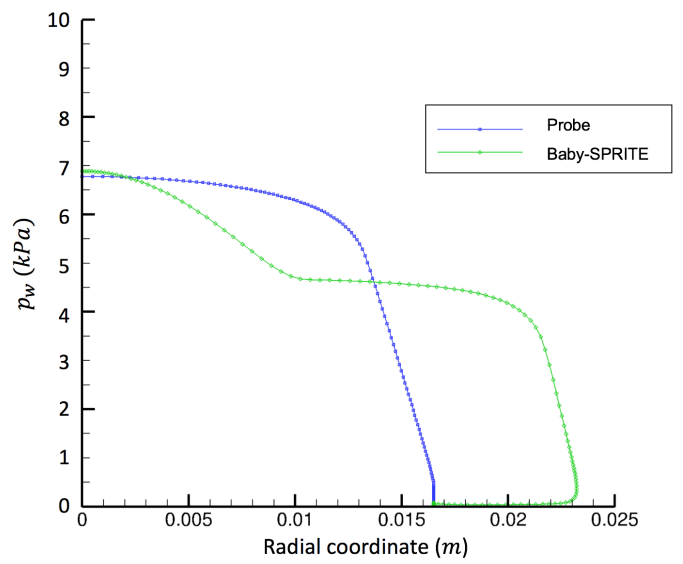

(b) Wall pressure $p_{w}(\mathrm{kPa})$

Figure 22: Heat flux and pressure distribution over the probe and the final baby-SPRITE in HyMETS. The probe solution is shown in blue, the baby-SPRITE is green. 


\section{Acknowledgments}

This work is supported by the NASA STMD Entry System Modeling Project (PM: Michael Wright; PI: Michael Barnhardt). Authors thank Jeffrey Hill, Dinesh Prabhu, Chun Tang, and David Saunders for helpful discussions.

\section{References}

${ }^{1}$ Prabhu, D. K., "Flowfield Analysis of a Small Entry Probe (SPRITE) Tested in Arc Jet", AIAA Paper 2012-0216, The 50th AIAA Aerospace Sciences Meeting, Nashville, Tennessee, January 2012.

${ }^{2}$ Yoon, S., Gnoffo, P. A., White, J. A., and Thomas, J. L., "Computational Challenges in Hypersonic Flow Simulations", AIAA Paper 2007-4265, The 39th AIAA Termophysics Conference, Miami, Florida, June 2007.

${ }^{3}$ Brune, A. J., Bruce, W. E., III, Glass, D. E., and Splinter, S. C., "Computational Simulations of the NASA Langley HyMETS Arc-Jet Facility", 2017 JANNAF - Interagency Propulsion Committee meeting, Newport News, Virginia, December 2017.

${ }^{4}$ Duzel, U., Schroeder, O., Zhang, H., and Martin, A., "Computational Prediction of NASA Langley HYMETS Arc Jet Flow with KATS", AIAA Paper 2018-1719, The AIAA SciTech Forum, Kissimmee, Florida, January 2018.

${ }^{5}$ Splinter, S. C., Bey, K. S., Gragg, J. G., and Brewer, A. R., "Comparative Measurements of Earth and Martian Entry Environments in the NASA Langley HYMETS Facility", AIAA Paper 2011-1014, The 49th AIAA Aerospace Sciences Meeting, Orlando, Florida, January 2011.

${ }^{6}$ Lachaud, J., Scoggins, J. B., Magin, T. E., Meyer, M. G., and N. N. Mansour, "A generic local thermal equilibrium model for porous reactive materials submitted to high temperatures", International Journal of Heat and Mass Transfer, Vol. 108, May 2017, pp. 1406-1417.

${ }^{7}$ Chan, W. M., Gomez, R. J., Rogers, S. E., Buning, P. G., "Best Practices in Overset Grid Generation", AIAA Paper 2002-3191, The 32nd AIAA Fluid Dynamics Conference, St. Louis, Missouri, June 2002.

${ }^{8}$ Wright, M. J, Candler, G. V., and Bose, D., "Data-Parallel Line Relaxation Method for the Navier-Stokes Equations", AIAA Journal, Vol. 36, September 1998, pp. 1603-1609.

${ }^{9}$ Saunders, D. A. and Prabhu, D. K., "BLAYER User Guide", NASA TM 2018 - 219749.

${ }^{10}$ Yoon, S. and Jameson, A., "Lower-Upper Symmetric Gauss-Seidel Method for the Euler and Navier-Stokes Equations", AIAA Journal, Vol. 26, September 1988, pp. 1025-1026.

${ }^{11}$ Shuen, J. S. and Yoon, S., "Numerical Study of Chemically Reacting Flows Using Lower-Upper Symmetric Successive Overrelaxation Scheme", AIAA Journal, Vol. 27, December 1989, pp. 1752-1760.

${ }^{12}$ Park, C. and Yoon, S., "Fully-Coupled Implicit Method for Thermo-Chemical Nonequilibrium Air at Sub-Orbital Flight Speeds", Journal of Spacecraft and Rockets, Vol. 28, No. 1, January-February 1991, pp. 31-39.

${ }^{13}$ Yoon, S., Barnhardt, M. D., and Candler, G. V., "Simulations of High-Speed Flow Over an Isolated Roughness", AIAA Paper 2010-1573, 48th AIAA Aerospace Sciences Meeting, Orlando, Florida, January 2010.

${ }^{14}$ Steger, J. L. and Warming, R. F., "Flux Vector Splitting of the Inviscid Gasdynamic Equations with Applications to Finite-Difference Methods", Journal of Computational Physics, Vol. 40, April 1981, pp. 263-293.

${ }^{15}$ Candler, G. V. and MacCormack, R. W., "The Computation of Hypersonic Ionized Flows in Chemical and Thermal Nonequilibrium", Journal of Thermohysics and Heat Transfer, Vol. 5, No. 3, 1991, pp. 266-273.

${ }^{16}$ Candler, G. V., Wright, M. J., and McDonald, J. D., "Data-Parallel Lower-Upper Relaxation Method for Reacting Flows", AIAA Journal, Vol. 32, December 1994, pp. 2380-2386.

${ }^{17}$ Wissink, A. M., Lyrintzis, A. S., and Strawn, R. C., "Parallelization of a Three-Dimensional Flow Solver for Euler Rotorcraft Aerodynamics Predictions", AIAA Journal, Vol. 34, November 1996, pp. 2276-2383.

${ }^{18}$ Yoon, S., Jameson, A., and Kwak, D., "Effect of Artificial Diffusion Schemes on Multigrid Convergence", AIAA Paper 95-1670, AIAA 12th Computational Fluid Dynamics Conference, San Diego, California, June 1995.

${ }^{19}$ Wright, M. J., Candler, G. V., and Prampolini, M., "Data-Parallel Lower-Upper Relaxation Method for the Navier-Stokes Equations", AIAA Journal, Vol. 34, July 1996, pp. 1371-1377.

${ }^{20}$ Park, C., "Assessment of Two-Temperature Kinetic Model for Ionizing Air", Journal of Thermophysics and Heat Transfer, Vol. 3, No. 3, 1989, pp. 233-244. 